\title{
Epilepsy: stigma and management from Pakistan's perspective
}

\begin{abstract}
Epilepsy is one of the chronic neurological disorders that have been an increasing burden to the developing countries. About 50million people worldwide suffer from epilepsy and approximately $90 \%$ of them are living in developing countries. According to WHO, 6 to 8 million people with epilepsy and 3 to 4 million (assuming a national average treatment gap of $50 \%$ ) remain untreated. In a poor resource country like Pakistan, epilepsy remains as a public health challenge. This study aimed to assess knowledge, attitude and practices for epilepsy and to identify needs of epileptic patients for management of epilepsy. A cross-sectional study is conducted in Karachi, Pakistan. Data is collected from the epileptic patients coming to the public health hospitals of Karachi for treatment and analyzed through SPSS version 17.0. A total 150 individuals were interviewed including $47 \%$ males and $53 \%$ females. Majority of them were under 20-29 age groups. The knowledge regarding epilepsy causation was minimal, 49.3\% respondents considered it as a curable disease while $40 \%$ respondents considered it as a contagious disease. Loss of Consciousness $(85 \%)$, Urine Incontinence $(70 \%)$, Salivation and Drooling (67\%) were reported to be the major presentations of epilepsy. The most common therapies used in the treatment of epilepsy, included faith healers $(80 \%)$, ayurvedic medications (57\%) and homeopathy (10\%) along with Modern drugs $(63 \%)$. Poor socio-economic conditions, lack of education, religious concepts and non-compliance from patient as well as from physicians were the main causes of treatment gaps. Awareness programs should focus on the acceptance of epileptic patients in the community not only to improve the quality of life of epileptic patients but also their families and for reducing stigmatization of epileptic patients. The main success factor for treatment of epilepsy lies in awareness and efficient and effective utilization of resources for epilepsy management.
\end{abstract}

Keywords: epilepsy, knowledge, management, socio demographics, practices, Pakistan
Volume I Issue I - 2014

\author{
Sayeeda Amber Sayed,' Raheel Naz,' Samia \\ Zaheer $^{3}$ \\ 'Research Consultant, University of Calgary, Canada \\ ${ }^{2}$ Department of Health Management, Institute of Business \\ Management, Pakistan \\ ${ }^{3}$ Department of Health Management, Institute of Business \\ Management, Pakistan
}

\begin{abstract}
Correspondence: Sayeeda Amber Sayed, Research Consultant University of Calgary, 303-I305 I5 Ave SW, Calgary, AB T3C0X8, Canada, Tel + 403 99| 4309,
\end{abstract}

Email sayeedaamber@hotmail.com

Received: November 23, 2014 | Published: December 18,2014

\section{Abbreviations:}

ETG, epilepsy treatment gap; ILAE, international league against epilepsy; IBE, international bureau for epilepsy; WHO, world health organization; CAM, complementary and alternative medicine

\section{Introduction}

Epilepsy is a medical condition that produces seizures affecting a variety of mental and physical functions. It is also called a seizure disorder. When a person has two or more unprovoked seizures, they are considered to have epilepsy. Seizures happen when clusters of nerve cells in the brain signal abnormally, which may briefly alter a person's consciousness, movements or actions. Even with medications and other treatments, many people continue to have seizures, which severely limit their school achievements, employment prospects and participation in all walks of life.

Epilepsy is the most common chronic neurological disorder, affecting approximately 50million people worldwide, out of them 40million are estimated to live in developing countries. ${ }^{2}$ Worldwide prevalence rate of epilepsy varies from 2.8 to 19.5 per 1000 of the general population and is more prevalent among children. The reported prevalence of active epilepsy in developing countries ranges from 5 to 10 per 1000 people. $^{3}$ The prevalence are high due lack of resources as well as awareness leading to Epilepsy treatment Gap (ETG). The ETG is defined as the difference between the number of people with active epilepsy (who may benefit from treatment) and the number whose seizures are being appropriately treated. International health agencies such as International League against Epilepsy (ILAE), International Bureau for Epilepsy (IBE) and World Health Organization (WHO) are important organizations that are focusing on the treatment and awareness of epilepsy in the general population. ${ }^{4}$

Religious beliefs, limited resources, lack of education, non availability and limited treatment, duration and cost of treatments, adjunctive therapies, suboptimal dose and side effects are the main challenges for not seeking and getting epilepsy treated properly. ${ }^{5}$ Psychological perceptions, superstitious beliefs are also one of the biggest challenges in managing epilepsy. Especially in Asian countries there is a need to create awareness regarding epilepsy as well as its management through effective programs to decrease the burden of disease and to improve the quality of life of patients. Patients suffering from chronic diseases like epilepsy often use complementary and alternative medicine (CAM), peers, hakims etc. as first-line treatment because of myths, superstitions and stigma attached to the disease. ${ }^{6}$

There are different treatments available for epilepsy. One study shows that Vitamin D has a very important role in management of epilepsy and in seizure control. 13 patients having pharmacoresistant epilepsy had their vitamin D (25-hydroxy vitamin D3) status determined. After 90 days treatment with vitamin D3supplementation there was significant seizure freedom rate of $40 \% .^{7}$ Psychological treatment can also help in reducing seizures frequency at night but depends on the associated occurrence of morning seizures as well as 
on the identification, types and number of seizure favoring factors, the type and duration of initial symptoms, the behavioral state at seizure onset, the type of epilepsy and the level of intelligence according to a study. ${ }^{8}$ Besides medication and psychological treatment other preventive measures are also essential since epilepsy hampers quality of life of patient and disturbs the whole family on a continuous basis. ${ }^{9}$

A study was conducted by university of North Carolina on 31 parents of epileptic patients regarding seizure history, frequency, type, medical services etc. The results showed that parents of epileptic patients or their family's needs support from the medical services or health organizations in terms of supportive programs and awareness campaigns. ${ }^{10}$ The objectives of this research study were to assess knowledge, attitude and practices of people with epilepsy and identify perceived needs of epileptic patients for management of epilepsy.

\section{Materials and methods}

A cross sectional study was conducted in March 2013 in Karachi, Pakistan. A total of 150 people with epilepsy aged 18 years and above were interviewed face to face with the help of a standardized questionnaire. The interviewers read the questions to the study participants and recorded responses on an answering sheet. Sociodemographic information i.e. age, gender, education and occupational status were collected along with knowledge, attitude and practices regarding epilepsy. The statistical analysis was performed using SPSS version 17.0 for Windows. Descriptive methods were mainly used to summarize the data.

Informed consent was sought from all participants of the study. The participants had the right to leave the study anytime or not to

Table I Demographic profile of study participants answer any of the study questions. Data were kept anonymous by assigning identification numbers to the participants.

\section{Results and discussion}

A total number of 150 respondents were interviewed out of which 71 were males and 79 were females (Table 1). The age of the participants ranged from 20 to 60 years with median ranging between 20 to 29 years. $83 \%$ participants had completed secondary schooling followed by $17 \%$ study participants who had only primary education. $54 \%$ were employed and $46 \%$ were unemployed. The reason of the high unemployment rate was reported to be discrimination for epileptic patients. Almost $34 \%$ were working in private companies, $19 \%$ were self-employed and $19 \%$ had government jobs. 28\% didn't respond to the question. Based on the income level of respondents, $25 \%$ were earning between PKR 20,000 to 29,999 and only $15 \%$ were making PKR 40,000 or more. It is important to note that $9 \%$ were in the lowest income group and had difficulty getting treatment and making ends meet. Regarding marital status $46 \%$ were married, out of which $24 \%$ were first degree relative.

$51 \%$ of the respondents had never heard about epilepsy before it had been diagnosed. This shows the lack of awareness regarding epilepsy in Pakistan. On further probing regarding knowledge about epilepsy, $56 \%$ reported epilepsy as a hereditary disorder while $43 \%$ responded that it is a brain disorder. $60 \%$ believed that the disease is caused by supernatural powers, followed by $55 \%$ respondents, who considered epilepsy is the results of sins of their patients/ancestors. $39 \%$ reflected epilepsy as contagious diseases whereas $26 \%$ agreed that it was caused due to blood transfusion and only $49 \%$ currently identified that epilepsy is a curable disease (Table 2).

\begin{tabular}{llll}
\hline Socio-demographics & n (\%) & Socio-demographics & n (\%) \\
\hline Gender & & Profession & \\
Male & $71(47)$ & Private Job & $51(34)$ \\
Female & $79(53)$ & Government Job & $29(19)$ \\
Age & & Business & $29(19)$ \\
$20-29$ & $46(31)$ & Income Range(PKR) & \\
$30-39$ & $41(27)$ & 10,000 to 19,999 & $14(9)$ \\
$40-49$ & $28(19)$ & 20,000 to 29,999 & $38(25)$ \\
$50-59$ & $22(15)$ & 30,000 to 39,999 & $24(16)$ \\
$60 \&$ above & $13(9)$ & 40,000 to 49,999 \\
Qualification & & 50,000 to 99,999 & $11(7)$ \\
Primary Education & $24(16)$ & Marital Status & $12(8)$ \\
Matriculation & $36(24)$ & Single & \\
Intermediate & $41(27)$ & Married & $64(43)$ \\
Graduation & $21(14)$ & Divorced/ Separated & $69(46)$ \\
Masters & $28(19)$ & Widow & $5(3)$ \\
Employment status & & Husband and Wife Relationship \\
Employed & $81(54)$ & 1st Degree Relative & $36(24)$ \\
Unemployed & $69(46)$ & 2nd Degree Relative & $19(13)$ \\
\hline
\end{tabular}


Table 2 Knowledge and attitude regarding epilepsy

\begin{tabular}{llll}
\hline Questions about epilepsy knowledge and attitude & Yes n (\%) & No n (\%) & Don't know n (\%) \\
\hline Before diagnosis have you heard about Epilepsy? & $74(49)$ & $76(51)$ & 0 \\
Is Epilepsy a Curable Disease? & $74(49)$ & $36(24)$ & $40(27)$ \\
Is Epilepsy a Brain Disorder? & $65(43)$ & $34(23)$ & $51(34)$ \\
Is Epilepsy a Hereditary Disorder? & $84(56)$ & $27(18)$ & $39(26)$ \\
Is Epilepsy due to Supernatural Powers? & $90(60)$ & $37(25)$ & $23(15)$ \\
Is Epilepsy due to Sins of Patients/ Ancestors? & $82(55)$ & $40(27)$ & $28(19)$ \\
Is Epilepsy Contagious? & $59(39)$ & $52(35)$ & $39(26)$ \\
Is Epilepsy because of Blood Transfusion? & $39(26)$ & $54(36)$ & $57(38)$ \\
Is branding useful in treatment of epilepsy? & $53(35)$ & $33(22)$ & $64(43)$ \\
Can a child with epilepsy play games? & $78(52)$ & $54(36)$ & $18(12)$ \\
Do you think people with epilepsy should be employed? & $89(59)$ & $30(20)$ & $31(21)$ \\
Should a person with epilepsy marry? & $101(67)$ & $34(23)$ & $15(10)$ \\
People with epilepsy have less chance of marriage? & $70(47)$ & $33(22)$ & $47(31)$ \\
Should people with epilepsy have children? & $93(62)$ & $17(11)$ & $40(27)$ \\
Should a female with epilepsy get pregnant? & $77(51)$ & $24(16)$ & $49(33)$ \\
In pregnancy there are complications due to epilepsy? & $52(35)$ & $46(31)$ & $52(35)$ \\
What are the complications during pregnancy? & & & \\
Missed Abortion & $45(30)$ & $37(25)$ & $68(45)$ \\
Teratogenicity & $39(26)$ & $42(28)$ & $69(46)$ \\
Extra Consultation & $34(23)$ & $39(26)$ & $77(51)$ \\
More care \& cost during and after pregnancy & $34(23)$ & $40(27)$ & $76(51)$ \\
\hline
\end{tabular}

Moreover, 52\% study participants agreed that epileptic child should play games. 59\% respondents believed that people with epilepsy have a right for employment whereas $40 \%$ disagreed for an equal right of employment which clearly shows that people with epilepsy are not accepted in the society and they are discriminated by the society. About $47 \%$ of the respondents reported that people with epilepsy have less chances of getting marriage whereas $38 \%$ of the respondents didn't agree that people with epilepsy should have children.

Regarding triggering factors, symptoms and treatment of epilepsy, the knowledge of respondents was very limited (Table 3). Major triggering factor for epilepsy reported were emotional disturbances $(66 \%)$, fever (58\%), flickering light (54\%) and $43 \%$ by hunger and food items. Majority of the participants (85\%) reported that the most common presentation of an epileptic patient is loss of consciousness due to which patient falls and bites his tongue. Other symptoms of epilepsy stated were tongue biting (81\%), urine incontinence (70\%) and salivation and drooling (67\%).

Treatment options for epilepsy included mainly faith healers (80\%), modern drugs $(63 \%)$ and ayurvedic drugs $(57 \%)$. Though majority of the participants had completed secondary school, the belief system on faith healers is stronger than medications. Almost all of them (95\%) were consulting a faith healer for the epilepsy treatment. Regarding the use of branded names for the epilepsy treatment, participants were least concerned about branding and branded products for the treatment of epilepsy. The practices queries regarding management of epilepsy show huge gaps (Table 4). In case of an epileptic fit 71 $\%$ reported smelling of a shoe. This is stated to be the most frequent and traditional practice. Other common practices were putting keys in hands $(50 \%)$ while $27 \%$ mentioned some other practices followed by their relatives, which included calling a faith healer. Only half of the participants mentioned going to the hospital in case of an epileptic fit.

While answering about discrimination, almost $60 \%$ study participants informed being discriminated by schoolmates $(59.3 \%)$, teachers $(48 \%)$, family $(44.7 \%)$, colleagues $(54 \%)$ and community $(50.7 \%)$ by mentioning less social participation and considering themselves as an easy target for humiliation due to epilepsy. When asked whether they will allow their 'normal' children (not suffering from epilepsy) to play with other children having epilepsy $47 \%$ responded negatively. Similarly, $56 \%$ respondents did not agree to disclose about epilepsy before their daughter and son's marriage. These findings are clearly associated with the taboos associated with epilepsy in Pakistan. 
Table 3 Triggering factors, symptoms and treatment options for epilepsy

\begin{tabular}{lll}
\hline & Yes n (\%) & No n (\%) \\
\hline Triggering Factors & & \\
\hline Emotional disturbance & $99(66)$ & $51(34)$ \\
Fever & $87(58)$ & $63(42)$ \\
Flickering Light & $87(58)$ & $68(45)$ \\
Hunger & $64(43)$ & $86(57)$ \\
Food \& Drinks & $65(43)$ & $85(57)$ \\
\hline Symptoms & & \\
\hline Loss of Consciousness & $127(85)$ & $23(15)$ \\
Urine Incontinence & $105(70)$ & $45(30)$ \\
Salivation and Drooling & $101(67)$ & $49(33)$ \\
Biting of the Tongue & $122(81)$ & $28(19)$ \\
\hline Treatment Options & & \\
\hline Modern Drugs & $95(63)$ & $53(37)$ \\
Ayurvedic Drugs & $84(57)$ & $64(43)$ \\
Faith Healers & $118(80)$ & $30(20)$ \\
\hline
\end{tabular}

Table 4 Practices regarding epilepsy

\begin{tabular}{llll}
\hline Practices & Yes n (\%) & No n (\%) & Don't know n (\%) \\
\hline During an epileptic attack will your family make you & & & \\
\hline Smell a Shoe & $107(71)$ & $43(29)$ & $0(0)$ \\
Put Keys in hand & $75(50)$ & $58(39)$ & $17(11)$ \\
Go to Hospital & $86(57)$ & $64(43)$ & $0(0)$ \\
Others & $41(27)$ & $109(73)$ & $0(0)$ \\
\hline Is a child or adult with epilepsy discriminated by: & & & $13(9)$ \\
\hline School Mates & $89(59)$ & $48(32)$ & $13(9)$ \\
Teachers & $72(48)$ & $65(43)$ & $11(7)$ \\
Family & $67(45)$ & $72(48)$ & $18(12)$ \\
Colleagues & $81(54)$ & $51(34)$ & $22(15)$ \\
Community & $76(51)$ & $52(35)$ & $16(11)$ \\
Will you allow your child to play with a child having epilepsy? & $64(43)$ & $70(47)$ & $35(23)$ \\
\hline Will you reveal about the epilepsy of your & & & $35(23)$ \\
\hline Daughter before marriage & $66(44)$ & $49(33)$ & $45(30)$ \\
Son before marriage & $70(47)$ & & \\
\hline
\end{tabular}

\section{Discussion}

\section{The stigmata of epilepsy}

Epilepsy is one of the chronic neurological disorders that affect people of all ages. Epilepsy accounts for $0.5 \%$ of the global burden of disease, a time-based measure that combines years of life lost due to premature mortality and time lived in states of less than full health. ${ }^{11}$ Around 50million people worldwide have epilepsy and approximately $90 \%$ of them live in developing countries. While epilepsy is an increasing burden in developing countries, it also poses a challenge for developed countries, which could only be managed by reducing stigma and strategic treatment. As per our study findings, epilepsy is a stigma in the Pakistani society. This argument is manifested by The Irish Epilepsy Association, which reported that $48 \%$ of people consider epilepsy as stigma for their life. ${ }^{12}$ There is a dire need to educate patients, their family and public regarding epilepsy. Negative attitude of public towards people with epilepsy adversely affects quality of life of patient as well as their families. ${ }^{11}$ Literature suggests that education and proper knowledge of epilepsy is required to overcome stigma 
of epilepsy that was previously ignored. ${ }^{13}$ Awareness is required regarding epilepsy otherwise it will remain as a stigma for the coming generations and if not controlled through modern treatment, unethical practices by quacks will be on rise.

\section{Misconception and discrimination}

There are lots of misconceptions associated with epilepsy. $80 \%$ study respondents reported that epilepsy is caused by supernatural power, which is also mentioned by Epilepsy foundation article. Throughout history, people with epilepsy and their families have suffered unfairly because of the ignorance of others. For centuries epilepsy was considered a curse of the Gods or worse that it was because of supernatural powers or sins of patients or ancestors. More than 100,000 women have been tortured and murdered believing that they are witches. ${ }^{14}$ The perception that epilepsy is contagious is discussed in a previous study that concludes the typical thinking that epilepsy could be transmitted through saliva. ${ }^{15}$ Because of limited knowledge respondents also believed that epilepsy is transmitted through blood transfusion or it is a hereditary disease. But as per epilepsy foundation, epilepsy is caused due to various reasons like brain tumors, stroke, head injuries etc. and it is not transmitted via blood transfusion or saliva. ${ }^{1}$ These misconception leads to a lot problems and aggravates the disease. ${ }^{16}$

Behavior and discrimination by family, teachers, friends \& community can further demoralize the patients (Table 4). People with epilepsy are afraid to disclose their condition to others because they are afraid that they will stop socializing with them or their children. ${ }^{11}$ According to Epilepsy Fact Sheet October 2012, until the 1970s, it was legal in United States to deny people with seizures access to restaurants, theatres and recreational centers and to other public buildings. ${ }^{9,11}$ According to another study, 29\% people with epilepsy did not feel comfortable to tell their friends and families regarding their disease in order to avoid change of attitude, $48 \%$ reported that their family and friends attitude changes when they know about their disease, while $48 \%$ were afraid of the word epilepsy. ${ }^{17}$

\section{Marriage and employment problems}

People with epilepsy face problems in their reproductive years as well as epileptic women during the pregnancy period. According to Epilepsy Foundation, nearly one-third of epileptic men suffer from erectile dysfunction and many epileptic women complain of dry vagina, painful contractions during sex, or low libido. ${ }^{18}$ Our study results are consistent with the fact that people with epilepsy finds difficult to get married and have children (Table 3). Moreover, many epileptic patients do not know whether they will have a child after marriage or if they will develop complications if they get married. ${ }^{19}$ People with epilepsy also have fewer chances of employment opportunities (Table 2) though people with controlled seizure are equally capable of all types of jobs and they have the same intelligence and abilities. ${ }^{20}$ According to questionnaire study on Knowledge and attitudes towards epilepsy in school children and University Students in Rome, Italy largest number of correct answers for nearly all the questionnaire item regarding epilepsy were came from University students. ${ }^{21}$

\section{Family support and awareness}

Epilepsy affects the life of patients not because of the disease symptoms but tremendously because of psychological distress, particularly those with low socioeconomic status, low social support from family, unmarried and uneducated. ${ }^{22,23}$ Epilepsy is a public health challenge and needs proper social support systems, awareness and disease monitoring among public and patients. ${ }^{17} \mathrm{~A}$ teen's survey concluded that half of the teens have not heard about Epilepsy, 28\% did not know that it is a mental disorder and most teens did not know what to do if someone has an epileptic attack. It is not only between patient and general public; health care workers' knowledge regarding epilepsy is also limited. To reduce the gap, health facilities have to create awareness to improve healthcare knowledge regarding epilepsy. ${ }^{13,18}$ To create mass awareness concerned authorities need to focus their efforts, build proper strategies and invest in this cause. Holistically to reduce stigmata of epilepsy we have to educate larger community, advocate programs at individual and societal level. ${ }^{24}$

Socio economic status creates a major hurdle for management of epilepsy especially in Pakistan since it is a resource poor country. Improved knowledge and good health infrastructure would facilitate epilepsy management. Our study and similar other studies show high correlation between epilepsy management and knowledge. It is essential to provide more resources, develop innovative tools and educational models to improve patient's compliance and practice. ${ }^{25}$ Such efforts would require further in-depth research on epileptic patient's knowledge, attitudes and practices and how they are interrelated. Epilepsy is a disability affecting the quality of life of patient ${ }^{26}$ because of severity and frequency of seizures however people with epilepsy can enjoy a healthy life with proper treatment and social support. ${ }^{2}$

\section{Conclusion}

Study respondents suffering from epilepsy know far too little about epilepsy. Most patients with epilepsy have religious beliefs and erroneous perception of the disease, which negatively influences epilepsy management and increases treatment gaps. Epilepsy is considered as a stigma in Pakistan and affects the quality of life of people with epilepsy. Information campaigns designed to improve the general public's knowledge of epilepsy should be encouraged at all educational levels so that future generations no longer consider epilepsy a stigma. Informational sessions with children in schools and universities can help in bringing a positive change to the future as the main success factor for treatment of epilepsy lies in awareness along with efficient and effective utilization of resources for epilepsy management. Our findings also provide a useful starting point for a future in-depth population-based survey on epilepsy.

\section{Acknowledgements}

RaheelNaz collected the data from the respondents and performed the analysis. SamiaZaheer provided useful comments during analysis and manuscript writing. Sayeeda Amber Sayed wrote the final manuscript and provided supervision in the designing, analysis and reporting of the data.

\section{Conflict of interest}

The author declares no conflict of interest.

\section{References}

1. Epilepsy Foundation. About epilepsy. 2013.

2. Mbuba CK, Ngugi AK, Newton CR, et al. The epilepsy treatment gap in developing countries: a systematic review of the magnitude, causes, and intervention strategies. Epilepsia. 2008;49(9):1491-1503. 
3. Amira S, Gaafar I, Abbasher H, et al. A study of knowledge, attitude practice towards epilepsy among relative of epileptic patients in Khartoum State. Sudanese Journal of Public Health. 2009;4(4):393-398.

4. Doughty J, Baker GA, Jacoby A, et al. Cross-cultural differences in levels of knowledge about epilepsy. Epilepsia. 2003;44(1):115-123.

5. Devi MG, Vijander S, Kiran B. Knowledge, attitude and practices among patients of epilepsy attending tertiary hospital in Delhi, India and a review of Indian studies. Neurology Asia. 2010;15(3):225-232.

6. Sangeeta S, Sukriti J, Suman K, et al. Quackery masquerading as complementary and alternative medicine (CAM) in Patients with epilepsy. Journal of Evidence-Based Complementary \& Alternative Medicine. 2007;12(2):139-143.

7. Hollo A, Clemens Z, Kamondi A, et al. Correction of vitamin D deficiency improves seizure control in epilepsy: a pilot study. Epilepsy Behav. 2012;24(1):131-133.

8. Muller B. Psychological approaches to the prevention and inhibition of nocturnal epileptic seizures: a meta-analysis of 70 case studies. Seizure. 2001;10(1):13-33.

9. Jacoby A. Stigma, epilepsy and quality of life. Epilepsy Behav. 2002;3(6S2):10-20.

10. Aytch LS, Hammond R, White C. Seizures in infants and young children an exploratory study of family experiences and needs for information and support. J Neurosci Nurs. 2001;33(5):278-285.

11. World Health Organization (WHO). Epilepsy. World Health Organization fact sheet; 2012

12. Brainwave -The Irish Epilepsy Association. Epilepsy \& Stigma Survey 2012.

13. Heijnders M, Van Der Meij S. The fight against stigma: an overview of stigma reduction strategies and interventions. Psychol Health Med. 2006;11(3):353-363.

14. Epilepsy Foundation. Facts about Seizures and Epilepsy. 2013.

15. Sandy F. Public education and awareness campaign on Epilepsy. Epilepsy Foundation; 2011.
16. Paschal AM, Hawley SR, Romain TS, et al. Epilepsy patients' perceptions about stigma, education, and awareness: Preliminary responses based on a community participatory approach. Epilepsy Behav. 2007;11(3):329-337.

17. Baker GA. People with epilepsy: what do they know and understand, and how does this contribute to their perceived level of stigma? Epilepsy Behav. 2002;396S2):26-32.

18. Harimanana A, Chivorakul P, Souvong V, et al. Is insufficient knowledge of epilepsy the reason for low levels of healthcare in the Lao PDR? BMC Health Serv Res. 2013;13:41.

19. Al-Adawi S, Al-Ismaily S, Martin R, et al. Psychosocial aspects of epilepsy in Oman: attitude of health personnel. Epilepsia. 2001;42(11):1476-1481.

20. Kobau R, Price P. Knowledge of epilepsy and familiarity with this disorder in the U.S. population: Results from the 2002 Health Styles survey. Epilepsia. 2003;44(11):1449-1454.

21. Mecarelli O, Voti PL, Vanacore N, et al. A questionnaire study on knowledge of and attitudes toward epilepsy in school children and university students in Rome, Italy. Seizure. 2007;16(4):313-319.

22. Young GB, Derry P, Hutchinson I, John V, Matijevic S, et al An epilepsy questionnaire study of knowledge and attitudes in Canadian college students. Epilepsia. 2002;43(6):652-658.

23. Hills MD, MacKenzie HC. New Zealand community attitudes toward people with epilepsy. Epilepsia. 2002;43(12):1583-1589.

24. Epilepsy awareness. Now I know! Facts about epilepsy-ending the myths. 2012.

25. Thacker AK, Verma AM, Ji R, et al. Knowledge awareness and attitude about epilepsy among school teachers in India. Seizure. 2008;17(8):684 690 .

26. Khalid A, Aslam N. Psychological distress among patients with epilepsy. Indian J Psychol Med. 2011;33(1):45-48. 